\title{
Correction to: Bio-Inspired Modular Relative Jacobian for Holistically Controlled Four-Arm Manipulators Using Opposite and Adjacent Dual-Arm Pairs
}

\author{
Rodrigo S. Jamisola Jr. ${ }^{1}$ (D) Rodney G. Roberts ${ }^{2}$
}

(c) King Fahd University of Petroleum \& Minerals 2021

\section{Correction to:}

\section{Arab J Sci Eng}

https://doi.org/10.1007/s13369-021-06046-z

In the original publication, Figs. 6 and 7 are published incorrectly. The correct figures are given below. The original publication has been updated of the same.

The original article can be found online at https://doi.org/10.1007/ s13369-021-06046-z.

\section{Rodrigo S. Jamisola Jr. \\ jamisolar@biust.ac.bw \\ Rodney G. Roberts \\ rroberts@fsu.edu}

1 Mechanical, Energy and Industrial Engineering Department, Botswana International University of Science and Technology, Palapye, Botswana

2 Electrical and Computer Engineering Department, Florida A\&M University - Florida State University, 2525 Pottsdamer Street, Tallahassee, FL 32310-6046, USA 

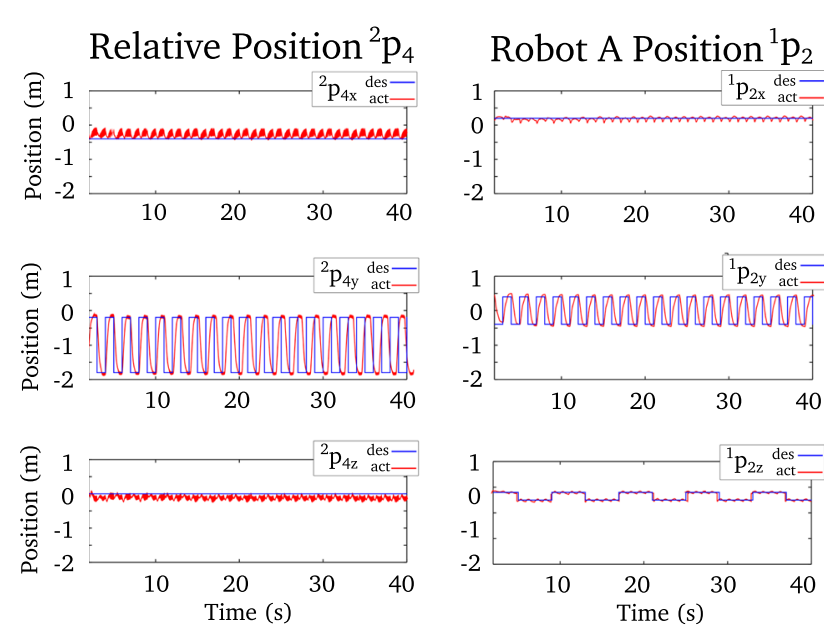

\section{Relative Position ${ }^{6} \mathrm{p}_{8}$}
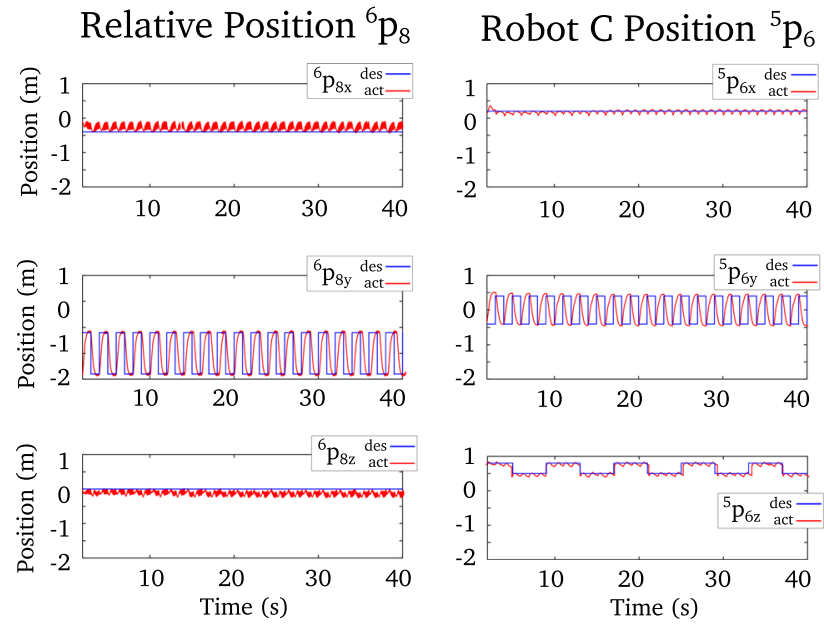

Fig. 6 Case 1 Pacing walking cycle results. The graphs show desired and actual values of the relative position of the front legs, ${ }^{2} \mathbf{p}_{4}$, and the hind legs, ${ }^{6} \mathbf{p}_{8}$, as well as the absolute position of Robot $A,{ }^{1} \mathbf{p}_{2}$, and Robot $C,{ }^{5} \mathbf{p}_{6}$. The front legs movement, ${ }^{2} \mathbf{p}_{4}$, is the main task, while ${ }^{1} \mathbf{p}_{2},{ }^{6} \mathbf{p}_{8}$, and ${ }^{5} \mathbf{p}_{6}$ are the secondary tasks in decreasing hierarchy
Position of Robot $\mathrm{A}{ }^{1} \mathrm{p}_{2}$
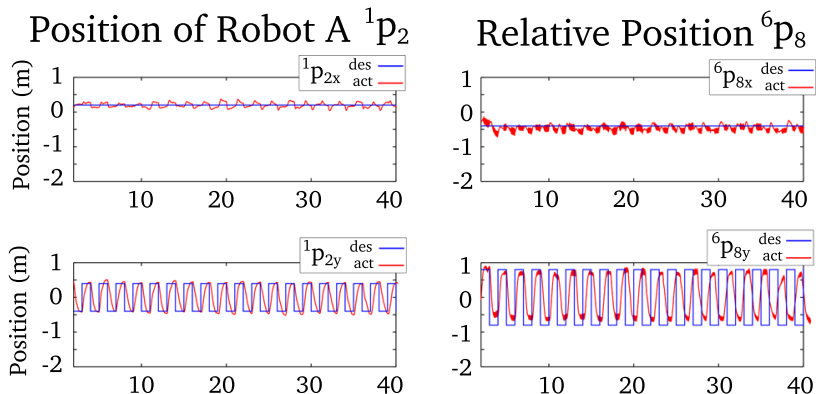

${ }^{6} \mathrm{p}_{8 \mathrm{y}}{ }_{\text {act }}^{\text {des }}$ -
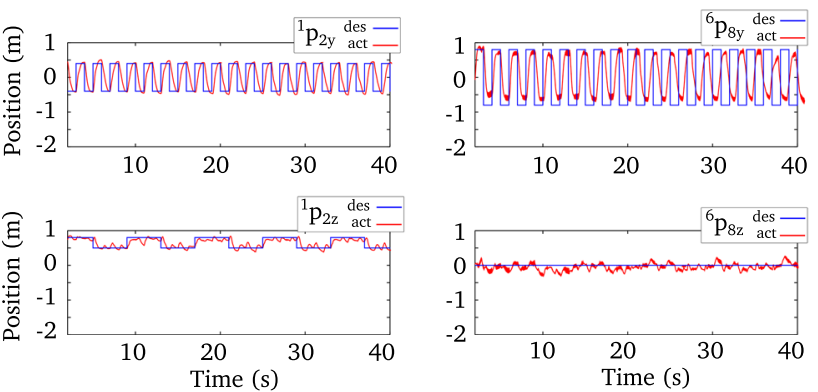

Position of Robot $\mathrm{A}{ }^{1} \mathrm{p}_{2}$

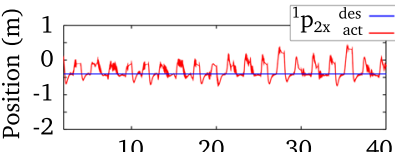

Relative Position ${ }^{6} \mathrm{p}_{8}$
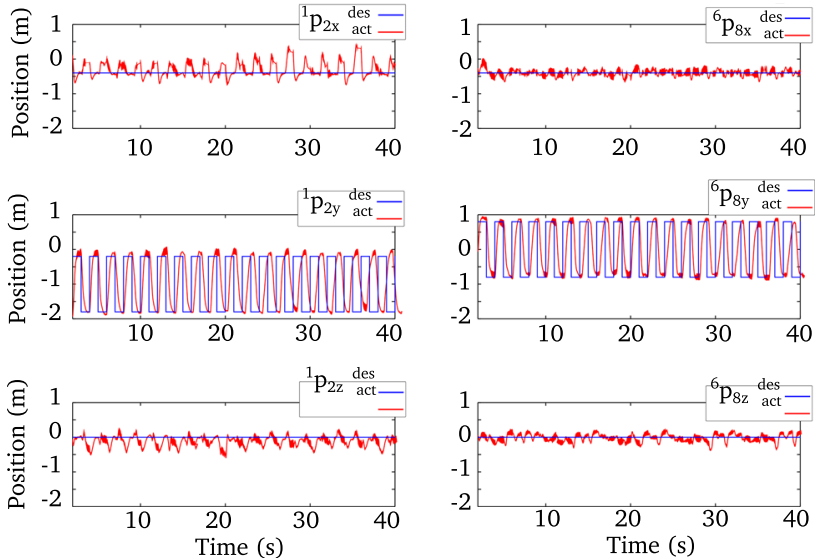

Fig. 7 Case 4 Counter-lateral leg pairing with equal null-space tasks hierarchy. The graphs show desired and actual values of counter-lateral leg pairing of Robot $A$ and Robot $C$ with the corresponding movement ${ }^{2} \mathbf{p}_{6}$ as the main task. The other legs movement now lies in the null space with equal hierarchy of task execution: front legs pair $^{2} \mathbf{p}_{4}$, Robot $A{ }^{1} \mathbf{p}_{2}$, and hind legs pair ${ }^{6} \mathbf{p}_{8}$ 